\title{
The medial prefrontal cortex is critical for memory retrieval and resolving interference
}

\author{
Gregory J. Peters, Christopher N. David, Madison D. Marcus, and David M. Smith ${ }^{1}$ \\ Department of Psychology, Cornell University, Ithaca, New York 14853, USA
}

\begin{abstract}
The prefrontal cortex (PFC) is known to be critically involved in strategy switching, attentional set shifting, and inhibition of prepotent responses. A central feature of this kind of behavioral flexibility is the ability to resolve conflicting response tendencies, suggesting a general role of the PFC in resolving interference. If so, the PFC should also be involved in memory retrieval, which involves competition between potential retrieval targets. Moreover, the PFC should be needed whenever interference is high, regardless of the strategic or attentional requirements of the task. To test this hypothesis, we temporarily inactivated the $\mathrm{mPFC}$ with muscimol and tested rats on several olfactory learning tasks. Rats given muscimol were able to learn a few discrimination problems when they were learned one at a time. However, they were severely impaired when they had to learn and remember many odors concurrently. Rats given muscimol also suffered greater interference when learning two lists of conflicting odor discrimination problems. Additionally, temporary mPFC inactivation during the acquisition of one set of odor memories actually improved the ability to learn a new set of conflicting odor memories. This paradoxical release from interference suggests that the MPFC plays an important role in acquiring and promoting the long term retrieval of memories. These results suggest that the mPFC plays a general role in resolving interference and that this is a key aspect of behavioral flexibility.
\end{abstract}

The prefrontal cortex (PFC) is thought to exert top-down influence on a variety of psychological processes, a function generally referred to as executive control (Shallice and Burgess 1996; Smith and Jonides 1999; Miller and Cohen 2001). Some processes known to involve the PFC include strategy selection (Monsell 2003; Block et al. 2007), directing attentional resources (Banich et al. 2000; Daffner et al. 2000; Asplund et al. 2010), and inhibiting prepotent behavioral responses (Aron et al. 2004; Chambers et al. 2006; Verbruggen and Logan 2008; Jonkman et al. 2009). Recently, a growing body of research has shown that the PFC is involved in similar functions in rodents. For example, medial prefrontal (mPFC) lesions cause impairments in a rodent adaptation of the Wisconsin card sorting task and the pattern of impairments is similar to those seen in humans (Ragozzino et al. 1999b; Birrell and Brown 2000; Ng et al. 2007). Like humans, the rats are unimpaired in learning the initial rule (e.g., respond according to one stimulus feature, such as odor), but they are severely impaired in learning to switch to a different stimulus feature (e.g., texture). Consistent with this, studies of spatial memory have shown that rats with mPFC damage are impaired in switching strategies from a place to a response strategy, although they are not impaired in reversals within a strategy, such as switching from a "go east" to a "go west" place strategy (Ragozzino et al. 1999a; Rich and Shapiro 2007). Other studies have also shown that reversal learning is not impaired by mPFC lesions (Aggleton et al. 1995; Floresco et al. 2008). A number of authors have also emphasized the mPFC role in attentional processes in rats (Birrell and Brown 2000; $\mathrm{Ng}$ et al. 2007). These studies demonstrate that the MPFC is critical in tasks that require rats to shift attention among competing stimulus features that indicate distinct behavioral responses. A common theme in this research is that the PFC contributes to behavioral flexibility, the ability to rapidly adopt new strategies, or behavioral response patterns (Block et al. 2007; Ragozzino 2007).

\footnotetext{
'Corresponding author

E-mail dms248@cornell.edu

Article is online at http://www.learnmem.org/cgi/doi/10.1101/lm.029249.112.
}

Although its role in strategy selection and attentional set shifting has been studied extensively, less is known about the PFC role in memory processes. However, recent imaging studies of human subjects have suggested that executive control processes also apply to the domain of memory retrieval. Interestingly, these studies have focused primarily on inhibition of retrieval. For example, when subjects are instructed to suppress the retrieval of some items from a study list, the ability to subsequently retrieve those items is impaired, and the PFC is active during retrieval suppression (Anderson and Green 2001; Depue et al. 2007). The PFC is also thought to play a role in another kind of memory inhibition called the retrieval-induced forgetting effect, in which practice with some items on a study list inhibits the subsequent retrieval of nonpracticed items, relative to a baseline condition (Anderson et al. 1994; Kuhl et al. 2007; Wimber et al. 2008). However, it is not clear from the human imaging data whether the PFC role is limited to the inhibition of retrieval or whether the PFC might also be involved in promoting the retrieval of target memories.

Recently, a number of investigators have begun to study the role of the PFC in memory processes in rodents. In these studies, the medial division of the PFC has been a primary target of interest because it is thought to be functionally similar to the dorsolateral PFC in primates (Uylings et al. 2003; Vertes 2006; Seamans et al. 2008), which is the region commonly found to be involved in memory processes in humans. In rodents, mPFC lesions impair various forms of spatial memory (Lee and Kesner 2003; Jo et al. 2007; Lee and Solivan 2008; Churchwell et al. 2010). The $\mathrm{mPFC}$ has also been shown to be involved in a number of nonspatial memory tasks, including fear conditioning and extinction (Sotres-Bayon and Quirk 2010), transitive inference (DeVito et al. 2010), and memory for sequential order (DeVito and Eichenbaum 2011). Although these studies clearly suggest that the mPFC is involved in typical rodent memory tasks, there is currently no consensus about the precise contribution of the PFC to memory encoding and retrieval processes.

In the present study, we tested the hypothesis that the mPFC is involved in resolving interference. The idea that the PFC is 
needed for resolving competing response tendencies is central to theories about executive function. Consistent with this idea, memory retrieval often involves a process of selecting from among many competing retrieval targets, particularly in high interference situations. Evidence from neuropsychological and neuroimaging studies supports this hypothesis (for review, see Blumenfeld and Ranganath 2007). Human subjects with PFC damage make more memory errors as study list length increases (Petrides and Milner 1982) and they are more susceptible to interference (Incisa della Rocchetta and Milner 1993; Smith et al. 1995). Increased susceptibility to interference has also been reported in rodents with PFC damage (Granon et al. 1994). Indeed, interference is a prominent characteristic of many classic PFC tasks.

We used a recently developed high interference olfactory learning task to assess the mPFC role in memory (Butterly et al. 2012; Law and Smith 2012). In this task, rats learn an initial list of eight concurrently trained odor discrimination problems. After learning the first list, the rats are trained on a new list of eight discrimination problems. In order to induce interference, half of the odors from the first list also appear on the second list with their predictive values reversed. In a series of experiments, we examined whether rats with temporary inactivation of the mPFC (prelimbic and infralimbic cortex) could acquire and remember the initial list of concurrently trained discrimination problems, whether they were more susceptible to interference than controls when learning a new conflicting list of discrimination problems, and, finally, whether mPFC involvement during the early stages of learning influences memory encoding and subsequent susceptibility to interference.

\section{Results}

\section{Experiment 1}

\section{Acquisition of the concurrent discrimination task}

Extensive evidence indicates that the mPFC is not needed for simple discrimination learning (Ragozzino et al. 1999b; Birrell and Brown 2000; Ragozzino et al. 2003; Ng et al. 2007). These studies suggested that the PFC is not needed for remembering which cues were associated with reward, but is instead only needed when the task requires behavioral flexibility, such as switching strategies or attentional set shifting. However, our hypothesis that the mPFC plays a key role in resolving interference suggests that the $\mathrm{MPFC}$ may be needed whenever subjects must manage many items in memory regardless of the specific task requirements. If so, the mPFC may be needed for discrimination learning when many problems must be learned and remembered at the same time.

We tested this hypothesis by giving control and muscimol rats ( $n=7$ per group) concurrent training on a single list of eight odor discrimination problems. On each trial, the rats were presented with two cups containing odorized digging medium. Within each discrimination problem, one of the odors was always associated with a buried reward and the other was not. Each training session involved eight trials with each of the eight problems presented in a randomized sequence. The rats were given infusions prior to each of the first three training sessions. The percentage of trials with a correct response was submitted to a repeated measures ANOVA with inactivation condition (saline or muscimol) as a between subjects factor and training session (the three infusion sessions) as the within subjects factor (Fig. 1A). This analysis showed that muscimol inactivation significantly impaired learning (main effect of inactivation, $F_{(1,12)}=11.83, P<0.01$ ). However, both groups showed evidence of learning (main effect of training session, $F_{(2,24)}=37.88, P<0.001$, but no interaction of the inactivation and training session factors, $F_{(2,24)}=1.85$, $P=0.18)$. Thus, the mPFC is important for discrimination learning when several problems must be acquired concurrently.

\section{Performance of the concurrent discrimination task}

Another prominent idea is that a key consequence of PFC damage is perseveration, wherein subjects are unable to abandon previously learned response tendencies when conditions change (Rubia et al. 2003; Verbruggen and Logan 2008; Jonkman et al. 2009). In contrast, our hypothesis that the mPFC plays a key role in resolving interference suggests that the mPFC may be needed whenever subjects need to manage many items in memory, regardless of whether memory failures take the form of perseveration or other kinds of errors. To test this hypothesis, we examined the effects of muscimol on performance of the well learned concurrent discrimination task.

After the early acquisition testing described above, the rats continued the concurrent discrimination training, without further infusions, until they reached a criterion of at least $90 \%$ correct for two consecutive sessions. Although they were initially impaired, rats that had been given muscimol during acquisition subsequently caught up to controls and their performance was equivalent during the criterial session $\left(t_{(12)}=-0.34, P=0.74\right)$. After achieving the criterion, the rats were given an additional four training sessions. The first three involved muscimol or saline infusions while the fourth session did not involve any infusions and served as a return to baseline check. Rats that had been given muscimol infusions during acquisition served as saline controls during asymptotic performance and the rats that received control infusions during acquisition were given muscimol during asymptotic performance. The percent correct data for the three infusion sessions were submitted to a repeated measures ANOVA with inactivation condition (saline or muscimol) as the between subjects factor and training session as the within subjects factor (Fig. 1B). Rats given muscimol were significantly impaired in performing the task (main effect of inactivation, $F_{(1,12)}=60.44, P<0.001$ ), but performance of the muscimol rats returned to normal levels on the fourth session, when no infusions were given $\left(t_{(10)}=-0.41, P=0.69\right)$.

\section{Acquisition of blocked discrimination problems}

The above results support our hypothesis that the mPFC is critical whenever subjects must manage many items in memory at the same time (i.e., concurrent discrimination). However, if this is correct then the mPFC should not be as important if rats were allowed to learn one discrimination problem at a time. To test this, we trained control and muscimol rats ( $n=6$ per group) on the same odor discrimination problems in a blocked fashion. For these rats, training was similar to the concurrent training condition, except that the discrimination problems were presented one at a time and the rats only moved on to a new problem after reaching 90\% correct on the current problem (Fig. 1C).

On average, muscimol rats were impaired in learning the discrimination problems (trials to the criterion, $t_{(10)}=3.93, P<$ 0.005; percent correct, $\left.t_{(10)}=-2.69, P<0.05\right)$. However, muscimol had no effect on learning the first discrimination problem (percent correct, $t_{(10)}=-0.22, P=0.83$; trials to criterion, $\left.t_{(10)}=1.24, P=0.24\right)$, replicating previous findings of intact learning for single discrimination problems (Birrell and Brown 2000; Ragozzino et al. 2003). In fact, although rats with temporary mPFC inactivation often performed poorly on some of the discrimination problems, they were typically able to learn several problems at a normal rate. For each rat, we separated the eight discrimination problems into the four best and four worst in terms of 
A

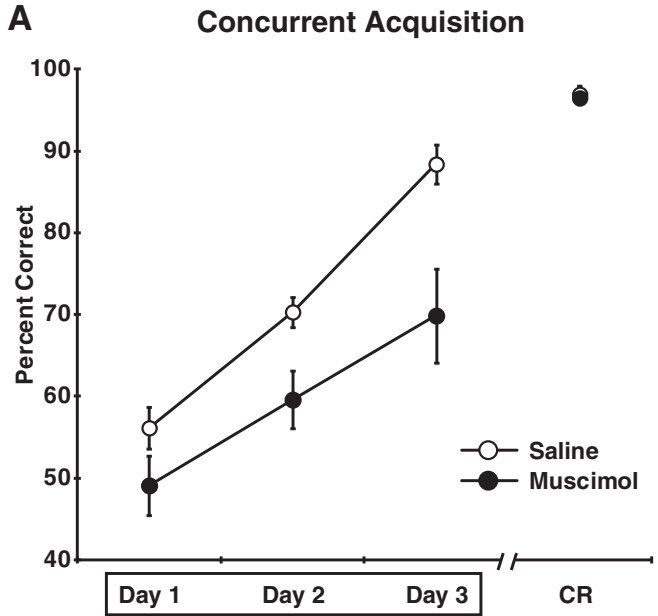

C

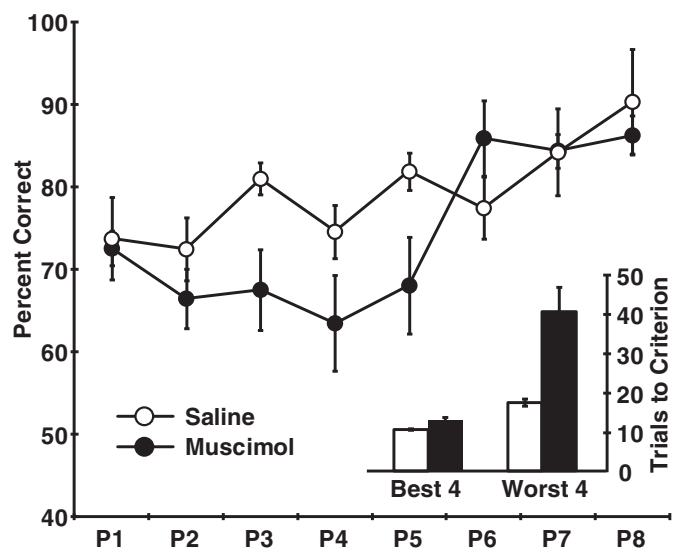

B

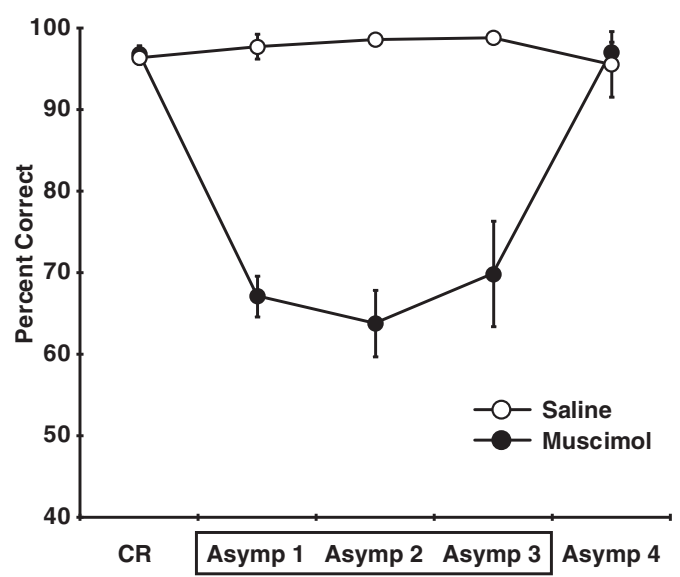

D

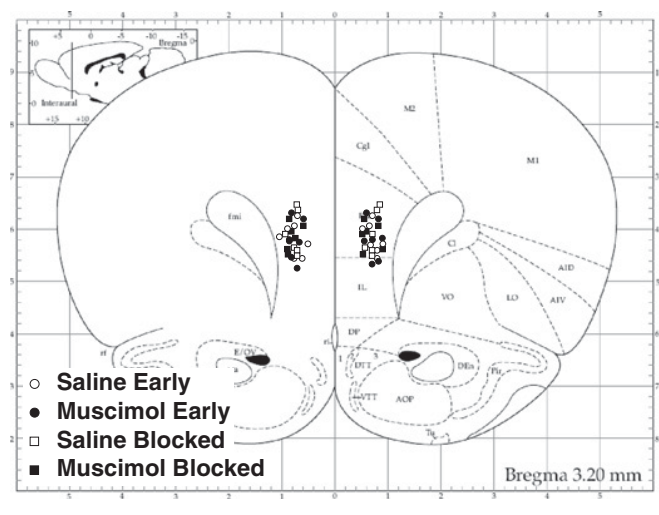

Figure 1. Experiment 1: Inactivation of the mPFC during concurrent and blocked odor discrimination. $(A)$ The percentage of trials with a correct response during the concurrent acquisition of eight odor discrimination problems is shown for saline and muscimol rats. Infusions were given prior to each of the first three training sessions (indicated by the box around days 1-3). By the time the rats reached the behavioral criterion (CR session), the muscimol rats had caught up to the performance level of the controls. (B) The effects of muscimol on asymptotic performance of the concurrent discrimination task are shown. Infusions were given prior to the first $3 \mathrm{~d}$ of post-criterial asymptotic performance (Asymp 1-3). Data of the criterial session (CR) and fourth session, which did not involve infusions, are also shown for comparison. Error bars are not visible for some values because the SEM was smaller than the area covered by the data markers. (C) The percentage of trials with a correct response over the course of training to the criterion on each of the eight odor discrimination problems (P1 - P8) is shown for the blocked training procedure. The average number of trials needed to reach the criterion for the best and worst problems for each rat is shown in the inset. For this experiment, the rats were given saline control infusions or muscimol infusions prior to each training session. $(D)$ Cannula placements are shown for the rats in Experiment 1, including the concurrent training condition (saline early and muscimol early) and the blocked training condition (saline blocked and muscimol blocked). (Atlas plates were adapted from Fig. 8 in Paxinos and Watson 1998, with permission from Elsevier (C) 1998.)

the number of trials needed to reach the criterion (Fig. 1C, inset). These data were submitted to a repeated measures ANOVA with inactivation condition (saline or muscimol) as a between subjects factor and problem type (best or worst problems) as a within subjects factor. This analysis revealed a significant interaction of the inactivation condition and problem type factors $\left(F_{(1,10)}=11.18\right.$, $P<0.01)$. The muscimol rats were severely impaired in learning their worst odor discrimination problems, requiring more than twice as many trials as the controls to learn each problem, but were relatively unimpaired on their best four problems (Fig. 1C, inset). Thus, rats given temporary mPFC inactivation were able to learn several discrimination problems as long as they learned them one at a time.

This pattern of results was not present in the rats trained in the concurrent condition described above. A similar analysis of the best four and worst four odor discrimination problems did not find an interaction of the inactivation condition and problem type factors $\left(F_{(1,12)}=2.68, P=0.13\right)$, indicating that although the muscimol rats were impaired in the concurrent task, as shown above, the impairment was not driven by very poor performance on some odor problems but normal performance on others. Instead, the magnitude of the impairment was similar for all odor discrimination problems in the concurrent condition.

\section{Experiment 2}

The finding that the $\mathrm{mPFC}$ is needed for the acquisition and retrieval of memory when many items are involved but not for only a few items provides suggestive evidence that the MPFC is involved in resolving interference. In the second experiment, we explicitly tested this hypothesis by training rats on two lists of odor discrimination problems that contain conflicting items. In 
this task, learning the first list causes significant proactive interference during learning of the second list (Butterly et al. 2012). However, the task does not require subjects to adopt a new strategy or shift attention from one stimulus feature to another. Thus, this experiment directly tests the hypothesis that interference alone, in the absence of strategic or attentional shifts, is sufficient to require mPFC involvement.

All of the rats ( $n=6$ per group) were first trained on list 1 exactly as in Experiment 1, except that there were no infusions, followed by training on a second list of eight odor discrimination problems in which each problem contained one novel odor paired with a previously presented odor from list 1 with its predictive value reversed. Half of the previously presented odors had been rewarded on the first list and half had not been rewarded. This ensured that the rats could not adopt a strategy of simply approaching the novel odors or avoiding the familiar odors. The rats were given muscimol or saline control infusions during the first three training sessions of the second list, followed by two additional sessions without infusions.

A previous study using this training procedure showed that temporary inactivation of the hippocampus selectively impaired the use of contextual information to resolve interference (Butterly et al. 2012). In that study, control rats performed better when they learned the two lists in different contexts, whereas rats with temporary hippocampal inactivation showed no contextual learning advantage. In order to determine whether the mPFC plays a similar role in the use of contextual information to overcome interference, half the rats were trained on the second list in the same context where they learned the first list and the other half were trained in a different context. This yielded a $2 \times 2$ design with the following groups: control-different context, controlsame context, muscimol-different context, and muscimol-same context.

Rats in the four groups reached the same level of asymptotic performance on the first list before beginning the second list $\left(F_{(3,20)}=0.33, P=0.81\right)$. The percent correct data from the list 2 training sessions were submitted to a two-way ANOVA with inactivation condition (saline or muscimol) and context condition (same or different context) as the between subjects factors and training session (five levels) as the within subjects factor (Fig. $2 \mathrm{~A})$. This analysis revealed a main effect of inactivation $\left(F_{(1,20)}=\right.$ 29.94, $P<0.001)$, an interaction of the inactivation and context condition factors $\left(F_{(1,20)}=6.62, P<0.05\right)$, and an interaction of the inactivation condition and session factors $\left(F_{(1,20)}=11.38\right.$, $P<0.001)$. The inactivation $\times$ context condition interaction was due to the fact that, as expected, control rats performed significantly better when they learned the two lists in separate contexts than when they learned the two lists in the same context (main effect of context condition for controls, $F_{(1,10)}=5.86, P<0.05$ ), but rats with muscimol inactivation did not (main effect of context condition for muscimol rats, $\left.F_{(1,10)}=1.66, P=0.23\right)$. The inactivation condition $\times$ session interaction was due to the fact that control rats showed significant improvement in performance during the saline infusion sessions (ANOVA of performance during the first three training sessions, $\left.F_{(2,22)}=38.81, P<0.0001\right)$ while muscimol rats showed little evidence of learning $\left(F_{(2,22)}=3.34\right.$, $P=0.07)$. Thus, unlike the effects of hippocampal inactivation, which was highly specific to the different context condition (Butterly et al. 2012), mPFC inactivation impaired performance regardless of the context condition.

Our experimental design also allowed us to directly assess interference in the control and muscimol subjects. If proactive interference occurred, performance should decline when subjects had to learn a second list after having learned the first list. Therefore, an interference index reflecting the change in performance across lists was computed for each subject (average percent
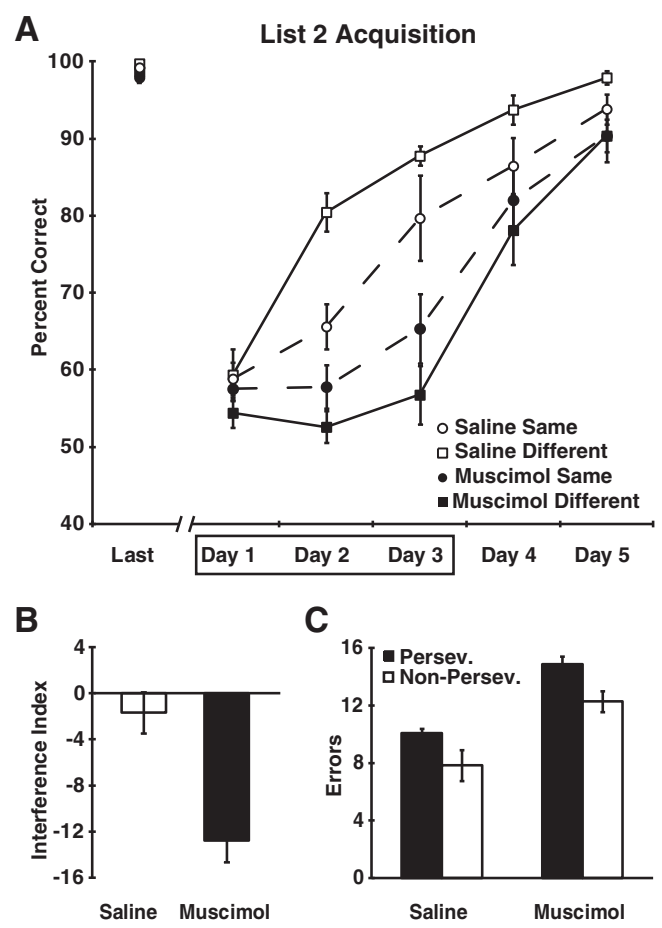

D

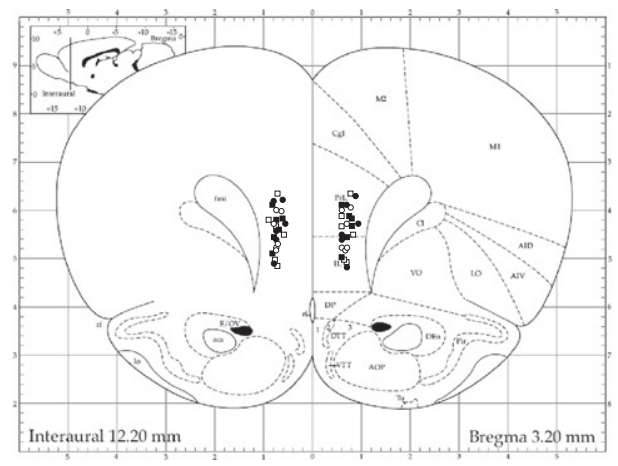

Figure 2. Experiment 2: Inactivation of the mPFC during learning of a second list of conflicting odor discrimination problems. ( $A$ ) Percent correct data are shown for the final day of training on list 1 (Last) and during the five training sessions of list 2, for control (open symbols) and muscimol (filled symbols) rats, and for the different context (squares) and same context (circles) rats. The rats were given infusions prior to the first three training sessions (indicated by the box). No infusions were given during the fourth and fifth sessions. $(B)$ The interference index, reflecting the decline in performance from list 1 to list 2, is shown for control and muscimol rats. (C) The numbers of perseverative (black bars) and nonperseverative (white bars) errors made during the infusion sessions are shown for control and muscimol rats. (D) Cannula placements are shown for the rats in Experiment 2 (symbols as in $A$ ). (Atlas plates were adapted from Fig. 8 in Paxinos and Watson 1998, with permission from Elsevier (C) 1998.)

correct on list 2 minus the average percent correct on list 1) and the interference scores of control and muscimol subjects were compared. Muscimol rats showed significantly greater interference than controls $\left(t_{(22)}=-4.31, P<0.001\right.$, Fig. $\left.2 \mathrm{~B}\right)$.

This task offers an additional test of the hypothesis that perseveration is the primary deficit in subjects with PFC damage. The second list presents the rats with two different kinds of problems. Half of the odor pairs contained a previously rewarded odor that was not currently rewarded and errors on these trials 
could be caused by perseverative responding (i.e., perseverative errors). The other half of the odor pairs did not contain a previously rewarded odor. Instead, these trials contained a previously nonrewarded odor and a novel odor. Errors on these trials would be nonperseverative errors. We asked whether rats with temporary mPFC inactivation were more prone to make perseverative errors than controls by submitting the number of errors to a repeated measures ANOVA with the inactivation condition as a between subjects factor and error type (perseverative or nonperseverative) as a within subjects factor (Fig. 2C). This analysis revealed a main effect of error type $\left(F_{(1,22)}=20.98, P<0.001\right)$, which indicated that all rats made more perseverative errors than nonperseverative errors, and a main effect of inactivation $\left(F_{(1,22)}=28.74, P<0.001\right)$, which confirmed that muscimol rats made more errors (of both types) than controls. However, there was no interaction of the error type and inactivation factors $\left(F_{(1,22)}=0.12, P=0.74\right)$, indicating that the muscimol rats did not make disproportionately more perseverative errors than controls.

\section{Experiment 3}

In Experiment 3, the long term effects of muscimol inactivation during the early stages of learning were assessed. Observations during pilot experiments suggested that temporary inactivation at the outset of learning list 1 could have an effect on the subsequent learning of conflicting information during list 2. Specifically, subjects that had temporary inactivation of the mPFC during the early stages of learning list 1 appeared to exhibit better performance (less interference) on list 2. If correct, this would suggest an mPFC role at the time of encoding which alters the way that the memories are subsequently retrieved. In order to formally assess this, rats ( $n=6$ per group) were given muscimol or control saline infusions prior to each of the first three sessions of list 1. They then continued daily training sessions until they reached the criterion, followed by four sessions of post-criterial training on list 1 . They were then given five training sessions on list 2 (Fig. $3)$. No infusions were given after the initial three sessions of list 1.

As in the first experiment, the rats that were given muscimol were impaired during the infusion sessions (Fig. 3A; main effect of inactivation, $\left.F_{(1,10)}=26.65, P<0.001\right)$. In this experiment, the muscimol rats showed only marginally significant evidence of learning (ANOVA of performance across the three infusion session, $\left.F_{(2,10)}=3.72, P=0.08\right)$. After the initial infusion sessions, the performance of rats with temporary inactivations caught up to that of controls and they performed equivalently during the post-criterial training sessions $\left(t_{(10)}=0.35, P=0.73\right)$. Importantly, both groups performed at very high levels for at least five sessions (the criterial session and the four post-criterial sessions, control mean $=99.11 \pm 0.15 \%$ correct, muscimol mean $=$ $98.96 \pm 0.31 \%$ correct, Fig. 3B). Remarkably, the rats that had been given muscimol infusions during the early sessions of list 1 performed significantly better on list $2\left(F_{(1,10)}=6.47, P<0.05\right.$, Fig. 3C). Thus, rats given early mPFC inactivation experienced less interference during list 2 , suggesting that memories encoded without the $\mathrm{mPFC}$ do not exert as much proactive interference as normally encoded memories.
A

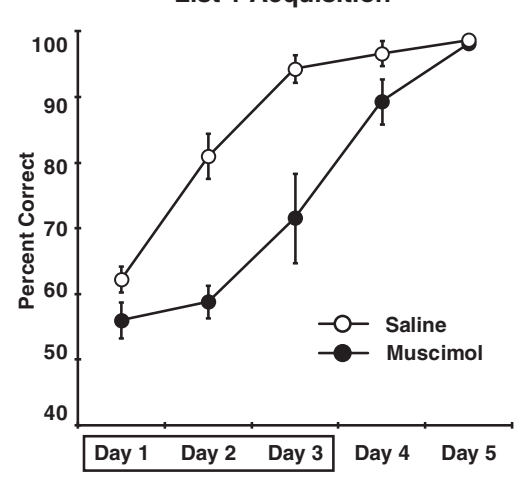

D

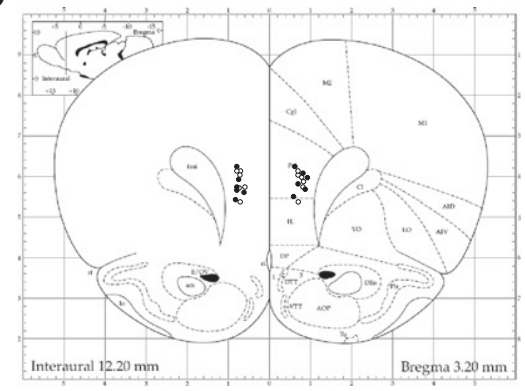

B

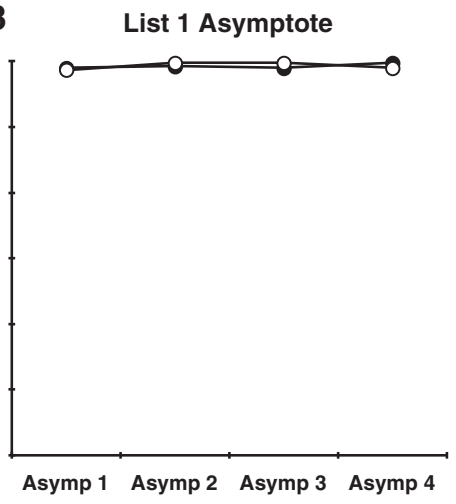

C

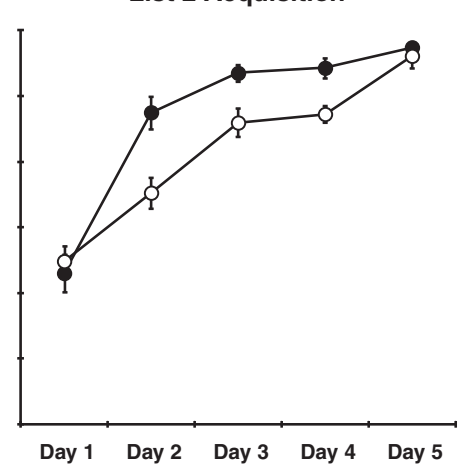

Figure 3. Experiment 3: Effects of early mPFC inactivation on subsequent performance. $(A)$ Percent correct for control and muscimol rats during the acquisition of the first list of odor discrimination problems. Infusions were given prior to the first three training sessions (indicated by the box), after which no additional infusions were given. As in Experiment 1, temporary inactivation of the mPFC impaired learning but performance caught up to that of controls by the fifth training session. (B) Both groups of rats performed at high levels for the four asymptotic performance sessions. Error bars are not visible for some values because the SEM was smaller than the area covered by the data markers. (C) Performance during learning of the second list of conflicting odor discrimination problems. Rats that had been given muscimol infusions during the early acquisition of the first list performed better than controls. ( $D$ ) Cannula placements are shown for the rats in Experiment 3 (symbols as in A). (Atlas plates were adapted from Fig. 8 in Paxinos and Watson 1998, with permission from Elsevier (C) 1998.) 


\section{Discussion}

Temporary inactivation of the mPFC impaired the acquisition and performance of the concurrent odor discrimination task, impaired the ability to learn a second list of conflicting odor associations after having learned the first list, and affected the long term retrieval of memories. Since the odor memories are probably not permanently stored in the $\mathrm{MPFC}$, these results are consistent with the idea that the PFC exerts top-down executive control over processing in other brain regions (Miller and Cohen 2001) and that PFC executive control applies to the domain of memory (Wagner et al. 2001; Reber et al. 2002; Badre and Wagner 2007). As discussed above, prominent theories of PFC function hold that the PFC is involved in strategy selection, attentional set shifting, and inhibiting prepotent response tendencies. Our results indicate that $\mathrm{mPFC}$ inactivation can produce severe deficits under conditions that do not require any of these functions. These results are consistent with the idea that the PFC is broadly involved in resolving interference (Badre and Wagner 2007; Blumenfeld and Ranganath 2007), regardless of whether the interference arises from competition among strategies, attentional targets, behavioral response tendencies, or memory retrieval targets.

Previous studies have shown that the MPFC is not needed for simple discrimination learning (Birrell and Brown 2000; Ragozzino et al. 2003). In our experiments, muscimol rats were impaired in the concurrent acquisition of many odor discrimination problems, although they were able to learn a few problems normally as long as they were learned one at a time (see Fig. 1C). Temporary inactivation of the $\mathrm{mPFC}$ also caused a striking impairment when infusions were given after the concurrent discrimination task was well learned, indicating that the mPFC role is not limited to learning but is also critical for retrieval. These results suggest that the mPFC is needed whenever subjects must simultaneously manage many items in memory, regardless of the particular type of task, and are consistent with an mPFC role in resolving interference. This role was directly confirmed in our second experiment, which was explicitly designed to induce high levels of interference (Butterly et al. 2012). Consistent with studies of human subjects (Incisa della Rocchetta and Milner 1993; Smith et al. 1995), muscimol rats were more susceptible to proactive interference than controls, resulting in severely impaired learning of the second list of conflicting odor discrimination problems.

Many authors have emphasized the idea that the PFC is critically involved in suppressing prepotent responses and that poor prefrontal control (e.g., in patients with lesions or children where PFC development is incomplete) results in perseveration (e.g., Tamm et al. 2002; Aron et al. 2003; Verbruggen and Logan 2008). Indeed, much of the work on executive control of memory processes in human subjects has focused on the inhibition of memory retrieval (Anderson and Green 2001; Depue et al. 2007). Our results indicate that the MPFC is not limited to inhibition, but is also involved in promoting the retrieval of desired responses and memories. The impaired performance of the well learned concurrent discrimination task could not have resulted from an inability to inhibit prepotent responses. In fact, continued responding in previously established ways (i.e., perseveration) would have supported excellent performance. The results of Experiment 2 also indicated that perseveration was not the primary problem in rats with temporary $\mathrm{mPFC}$ inactivation. When they were asked to learn a new list of conflicting items, muscimol rats made many more errors than controls. However, they did not make disproportionately more perseverative errors (erroneous responses to previously rewarded odors) than nonperseverative errors (erroneous responses to novel odors, Fig. 2C).

In Experiment 3, rats given muscimol during the initial stages of learning were subsequently able to learn a new list of conflict- ing items faster than controls. This counterintuitive result is also consistent with the idea of an MPFC role in promoting memory retrieval. Although all of the rats learned the first list to equivalently high levels, intact controls experienced greater proactive interference on the second list than the rats given muscimol during the early learning sessions (see Fig. 3). Apparently, having a functioning $\mathrm{mPFC}$ at the time of encoding results in memories that are particularly easy to retrieve in the future, and persistent intrusions of these old memories interferes with the subsequent acquisition of new conflicting memories. Consistent with this idea, human imaging studies have shown PFC activity during the successful encoding of memories (Reber et al. 2002; Ranganath et al. 2005; Spaniol et al. 2009). These findings suggest that the mPFC plays a critical role in the encoding and long term retrieval of important memories. In the present study, this lasted for several days, with the average time from the last muscimol infusion to the beginning of list 2 training being more than $6 \mathrm{~d}$.

Exactly how the mPFC promotes the long term retrieval of memories is not clear. During the initial stages of learning, as the odor memories are gradually laid down in their permanent storage site, mPFC neurons may become connected with the memory representations, possibly through interactions with the hippocampus (Anderson and Green 2001; Wagner et al. 2001). Later, retrieval of the memories could be triggered by any of several cues (e.g., return to the training context, presentation of one of the odor cues, etc.). However, the mPFC connections could serve as an additional source of excitatory input that primes the selective retrieval of the correct target memories. Neural network models suggest that this kind of top-down excitatory input, combined with diffuse inhibition of other nontarget representations, is an effective mechanism for resolving retrieval competition (Munakata et al. 2011).

This interpretation is consistent with each of our main findings. Learning a small number of discrimination problems in a blocked fashion is less sensitive to MPFC damage because there is little retrieval competition and non-PFC dependent sources of excitation (e.g., simple odor-reward associations) are sufficient to support performance. In the concurrent discrimination task, inactivation of the mPFC slowed acquisition because the presence of many partially encoded memories creates competition and one source of excitatory input to the correct memory targets is missing. Likewise, inactivation of the MPFC impaired asymptotic performance of the concurrent discrimination task because a key mechanism of resolving retrieval competition has been lost. In our third experiment, temporary mPFC inactivation during acquisition prevented the formation of the excitatory connections from the mPFC, resulting in weaker long term retrieval of the memories and reduced capacity for them to cause proactive interference. Together, these findings support the hypothesis that the mPFC is critically involved in modulating memory retrieval processes.

There is ample evidence from studies of humans and animals that the mPFC and hippocampus interact during learning and memory processes (Lee and Kesner 2003; Bunge et al. 2004; Wiltgen et al. 2004; Siapas et al. 2005; Anderson and Levy 2009) and there are anatomical connections between the two structures (Swanson 1981; Ferino et al. 1987; Hoover and Vertes 2007). Several authors have specifically proposed that the PFC exerts top-down control of memory processes by influencing hippocampal retrieval processes (Bunge et al. 2004; Anderson and Levy 2009; Munakata et al. 2011). Hippocampal neurons reliably respond to odor cues in similar tasks, suggesting that the hippocampus encodes odor memories (Wood et al. 1999; Manns et al. 2007) and a recent study of conditional discrimination found that temporary inactivation of the mPFC impaired memory and caused hippocampal responses to become less selective for particular odors (Navawongse and Eichenbaum 2013). This result is 
clearly consistent with the idea that mPFC input promotes the selective retrieval of specific odor memories via interactions with the hippocampus.

Previously, we showed that the hippocampus is critically involved in the olfactory memory task used here (Butterly et al. 2012). Using the same procedures as in Experiment 2, we found that temporary inactivation of the hippocampus selectively blocked the ability to use contextual information to overcome interference but had no effect on learning the second list in the same context. In contrast, temporary inactivation of the mPFC impaired learning regardless of the context condition (Fig. 2A). These findings suggest that both structures are involved in resolving interference, but in different ways. An extensive literature has shown that the hippocampus is involved in encoding contexts (for reviews, see Hirsh 1974; Wilson et al. 1995; Smith and Mizumori 2006) and we have suggested that associations of learned items with the learning context provides an automatic mechanism for interference-free retrieval of the relevant memories whenever subjects revisit the familiar context (Butterly et al. 2012). However, this mechanism is only useful when the items to be remembered are strongly linked to a particular context. In other conditions, a more general interference resolution mechanism must be used. As described above, the mPFC seems to fulfill this need by exerting a top-down influence on memory retrieval whenever there is retrieval competition.

The idea of conflict resolution is central to many theories of PFC function and, as other authors have noted (Badre and Wagner 2007; Blumenfeld and Ranganath 2007), a PFC role in resolving interference is consistent with that idea. This suggests that resolving mnemonic interference may be one facet of a broader conflict resolution function of the PFC. However, it is also possible that the classic deficits associated with PFC damage may reflect a failure to retrieve the appropriate strategy, attentional target, or behavioral response from memory due to poor interference resolution. The present results suggest that interference is sufficient to engage the PFC and studies of PFC interactions with the hippocampus may help determine the degree to which various kinds of conflict resolution may have mnemonic components (Lee and Solivan 2008; Anderson and Levy 2009; Navawongse and Eichenbaum 2013). In any case, we suggest that prefrontal modulation of retrieval processes is an important mechanism for successfully resolving competition and that this is a key aspect of behavioral flexibility.

\section{Materials and Methods}

\section{Subjects}

Sixty-two adult male Long-Evans rats (Charles River Laboratories, Wilmington, MA) were individually housed and maintained on a 12 -h light/dark cycle. Rats were food restricted to $80 \%-85 \%$ of their ad libitum weight, and were given free access to water. All experiments were conducted in compliance with guidelines established by the Cornell University Animal Care and Use Committee.

\section{Surgery and microinfusions}

Subjects were anesthetized with isoflurane and placed in a stereotaxic device (Kopf Instruments). The skull was exposed, bilateral craniotomies were drilled, and dual (bilateral) 22-gauge guide cannulae (Plastics One) were implanted using standard stereotaxic techniques. The guide cannulae were implanted so that infusion cannulae (22 gauge), which protruded $0.5 \mathrm{~mm}$ beyond the tip of the guide cannulae, were positioned in the prelimbic/infralimbic cortex (3.2 $\mathrm{mm}$ anterior and $0.5 \mathrm{~mm}$ lateral to bregma, and 2.7 $\mathrm{mm}$ ventral to the cortical surface). The guide cannulae were secured to the skull with bone screws and dental acrylic. Rats were allowed to recover for 5-10 d before beginning behavioral training. Temporary lesions were induced with the $\mathrm{GABA}_{\mathrm{A}}$ agonist muscimol. Thirty minutes prior to the relevant training sessions, $0.5 \mu \mathrm{L}$ of a solution containing $1 \mathrm{mg} / \mathrm{mL}$ muscimol or an equivalent volume of saline solution was infused into each hemisphere. The infusion cannulae were left in place for $1 \mathrm{~min}$ after the infusions.

\section{Apparatus}

Details of the apparatus, odor stimuli, and training procedures are given elsewhere (Butterly et al. 2012). Briefly, the rats were trained in Plexiglas chambers $(45 \mathrm{~cm}$ wide $\times 60 \mathrm{~cm}$ long $\times 40$ $\mathrm{cm}$ deep) equipped with a removable divider, which separated the odor presentation area from an inter-trial interval area. Thirty-two pure odorants served as cues. The amount of each odorant was calculated to produce an equivalent vapor phase partial pressure when mixed with $50 \mathrm{~mL}$ of mineral oil (Cleland et al. 2002). These odorants were mixed into corncob bedding material and presented to the rats in ceramic cups $(8.25 \mathrm{~cm}$ diameter, $4.5 \mathrm{~cm}$ deep) that fit into circular cutouts cemented to the floor of the chamber.

\section{Behavioral training procedures}

Prior to training, all of the rats were acclimated to the training apparatus (two 15-min sessions in each of the two contexts, described below) and shaped to dig in cups of scented bedding material for buried rewards (45-mg sucrose pellets, Bio-Serv).

\section{Experiment 1: Methods}

The first experiment was designed to assess the role of the mPFC in the acquisition and performance of a multi-problem concurrent discrimination task. The rats were given concurrent training on a list of eight odor discrimination problems (16 individual odors). The two odors comprising each problem were always presented together, one odor in each cup. Within each discrimination problem, one odor was always rewarded and the other was not. At the start of each trial, the experimenter placed the two cups containing the odorized bedding in the apparatus, raised the divider, and allowed the rat to dig until he retrieved the reward (corrections were always allowed following errors). A digging response was recorded if the rat displaced any of the bedding, except for incidental contact (e.g., stepping into the cup without digging). After consuming the reward, the rat was returned to the waiting area and the divider was replaced for an inter-trial interval of approximately $15 \mathrm{sec}$ while the experimenter prepared the cups for the next trial. The predictive value of the odors (rewarded or nonrewarded) was counterbalanced across subjects and their locations (left or right side of the chamber) were randomized. The rats were given daily training sessions consisting of 64 trials (eight trials with each discrimination problem, presented in an unpredictable sequence) until they reached a behavioral criterion of at least $90 \%$ correct on two consecutive sessions. After achieving this criterion, the rats were given an additional four post-criterial training sessions. Rats were given muscimol or saline control infusions during the first three training sessions (acquisition) and during the first three post-criterial sessions (asymptotic performance). The same cohort of rats was used in both tests. The rats that had been given muscimol infusions during acquisition served as saline controls during asymptotic performance and the rats that received control infusions during acquisition were given muscimol during asymptotic performance.

In order to determine whether the mPFC was needed for learning individual discrimination problems one at a time, two additional groups of rats (control and muscimol) were trained in a blocked learning condition. For these rats, the same eight odor discrimination problems were used, but each problem was presented repeatedly until the rat reached the criterion before moving on to the next problem. In order to match the concurrent training condition as closely as possible, we used a criterion of $90 \%$ correct over 10 trials and the rats were allowed to complete as many problems as possible within any given session, except 
that no new problems were begun after the 64th trial (i.e., the number of trials per session in the concurrent condition). In most cases, each session continued until the rat achieved the criterion for the last problem of the day and training on the next problem of the list began on the following day. In a few cases, the rat failed to reach the criterion on the last problem after 20 trials and training was discontinued for the day but resumed with the same problem on the following day. Daily training sessions were given until the rat achieved the criterion for all eight of the discrimination problems. None of the rats failed to reach the criterion for any of the problems. For consistency with the analysis of the concurrent training data, we computed the percentage of trials with a correct response over the course of training to the criterion on each of the discrimination problems. We also analyzed the number of trials needed to reach the criterion for each problem.

\section{Experiment 2: Methods}

Experiment 2 involved learning two lists of odor discrimination problems with conflicting items in order to assess the role of the mPFC in resolving interference. For this experiment, rats were trained on the first list of discrimination problems as described above, but with no infusions. After achieving the criterion and completing the four post-criterial training sessions (as in Experiment 1) the rats were trained on a second list of eight discrimination problems. Training on the second list was identical to the list 1 training sessions. However, in order to induce interference, each new discrimination problem consisted of a novel odor and an odor which had previously been presented on list 1 . Thus, list 2 consisted of 16 odors, half of which had appeared previously on list 1 . Of the eight odors taken from list 1 , half had been rewarded previously and half had not. For example, if the first two odor pairs on list 1 were $A+/ B-$ and $\mathrm{C}+/ \mathrm{D}-$, the first two odor pairs on list 2 would be $\mathrm{X}+/ \mathrm{A}-$ and $\mathrm{D}+/ \mathrm{Y}-$. This ensured that the rats could not adopt a strategy of simply approaching or avoiding the novel odor within each new odor pair.

Experiment 2 also involved a context manipulation in which half the rats were trained on the second list in the same context where they learned the first list (a white box), while the other half learned the second list in a new context (a black box). The two contexts differed along the following dimensions: color of the chamber (white or black), color of the curtains surrounding the training area (black or white), substrate in the chamber (Plexiglass floor or a black rubber mat), the $65-\mathrm{dB}$ continuous background masking noise (white noise or pink noise), and the ambient odor left by wiping out the chamber with baby wipes prior to each training session (unscented or scented, Rite Aid, Inc). Additionally, the rats were transported in covered cages to the experimental area by different methods for the two contexts (via a cart or carried by hand). All of the rats were given five training sessions on the second list. The rats were given muscimol or saline control infusions prior to the first three training sessions. No infusions were given during the final two training sessions.

\section{Experiment 3: Methods}

In Experiment 3, rats were trained on the two odor lists as in Experiment 2. However, the rats were given muscimol or control saline infusions prior to the first three sessions of list 1, after which no more infusions were given. Daily training continued until they reached the criterion and had completed four additional postcriterial training sessions. This was followed by five training sessions on list 2 in the same context.

\section{Data analysis}

For each subject and training session, the percentage of trials with a correct response and other dependent measures (e.g., trials to criterion, numbers of errors of various types, etc.) were computed and the data were submitted to ANOVA (SPSS, IBM Inc.). For all re- peated measures analyses, Hyunh-Feldt corrected $P$-values were automatically computed in order to adjust for violations of the sphericity assumption.

\section{Histology}

Following testing, all rats were anesthetized with isoflurane, transcardially perfused with $10 \%$ paraformaldehyde, and their brains removed, frozen, and sectioned at $40 \mu \mathrm{m}$, mounted on slides, and stained with cresyl violet in order to identify the infusion locations.

\section{Acknowledgments}

This work was supported by NIMH grant MH083809 to D.M.S.

\section{References}

Aggleton JP, Neave N, Nagle S, Sahgal A. 1995. A comparison of the effects of medial prefrontal, cingulate cortex, and cingulum bundle lesions on tests of spatial memory: Evidence of a double dissociation between frontal and cingulum bundle contributions. J Neurosci 15: 7270-7281.

Anderson MC, Green C. 2001. Suppressing unwanted memories by executive control. Nature 410: 366-369.

Anderson MC, Levy BJ. 2009. Suppressing unwanted memories. Curr Dir Psychol Sci 18: 189-194.

Anderson MC, Bjork RA, Bjork EL. 1994. Remembering can cause forgetting: Retrieval dynamics in long-term memory. J Exp Psychol 20: 1063-1087.

Aron AR, Fletcher PC, Bullmore ET, Sahakian BJ, Robbins TW. 2003. Stop-signal inhibition disrupted by damage to right inferior frontal gyrus in humans. Nat Neurosci 6: 115-116.

Aron AR, Robbins TW, Poldrack RA. 2004. Inhibition and the right inferior frontal cortex. Trends Cogn Sci 8: 170-177.

Asplund CL, Todd JJ, Snyder AP, Marois R. 2010. A central role for the lateral prefrontal cortex in goal-directed and stimulus-driven attention. Nat Neurosci 13: 507-512.

Badre D, Wagner AD. 2007. Left ventrolateral prefrontal cortex and the cognitive control of memory. Neuropsychologia 45: 2883-2901.

Banich MT, Milham MP, Atchley RA, Cohen NJ, Webb A, Wszalek T, Kramer AF, Liang Z, Barad V, Gullett D, et al. 2000. Prefrontal regions play a predominant role in imposing an attentional 'set': Evidence from fMRI. Brain Res Cogn Brain Res 10: 1-9.

Birrell JM, Brown VJ. 2000. Medial frontal cortex mediates perceptual attentional set shifting in the rat. J Neurosci 20: 4320-4324.

Block AE, Dhanji H, Thompson-Tardif SF, Floresco SB. 2007. Thalamicprefrontal cortical-ventral striatal circuitry mediates dissociable components of strategy set shifting. Cereb Cortex 17: 1625-1636.

Blumenfeld RS, Ranganath C. 2007. Prefrontal cortex and long-term memory encoding: An integrative review of findings from neuropsychology and neuroimaging. Neuroscientist 13: 280-291.

Bunge SA, Burrows B, Wagner AD. 2004. Prefrontal and hippocampal contributions to visual associative recognition: Interactions between cognitive control and episodic retrieval. Brain Cogn 56: 141-152.

Butterly DA, Petroccione MA, Smith DM. 2012. Hippocampal context processing is critical for interference free recall of odor memories in rats. Hippocampus 22: 906-913.

Chambers CD, Bellgrove MA, Stokes MG, Henderson TR, Garavan H, Robertson IH, Morris AP, Mattingley JB. 2006. Executive "brake failure" following deactivation of human frontal lobe. J Cogn Neurosci 18: $444-455$.

Churchwell JC, Morris AM, Musso ND, Kesner RP. 2010. Prefrontal and hippocampal contributions to encoding and retrieval of spatial memory. Neurobiol Learn Mem 93: 415-421.

Cleland TA, Morse A, Yue EL, Linster C. 2002. Behavioral models of odor similarity. Behav Neurosci 116: 222-231.

Daffner KR, Mesulam MM, Scinto LF, Acar D, Calvo V, Faust R, Chabrerie A, Kennedy B, Holcomb P. 2000. The central role of the prefrontal cortex in directing attention to novel events. Brain 123 (Pt 5): 927-939.

Depue BE, Curran T, Banich MT. 2007. Prefrontal regions orchestrate suppression of emotional memories via a two-phase process. Science 317: $215-219$.

DeVito LM, Eichenbaum H. 2011. Memory for the order of events in specific sequences: Contributions of the hippocampus and medial prefrontal cortex. J Neurosci 31: 3169-3175.

DeVito LM, Lykken C, Kanter BR, Eichenbaum H. 2010. Prefrontal cortex: Role in acquisition of overlapping associations and transitive inference. Learn Mem 17: 161-167. 
Ferino F, Thierry AM, Glowinski J. 1987. Anatomical and electrophysiological evidence for a direct projection from Ammon's horn to the medial prefrontal cortex in the rat.ExpBrain Res 65: 421-426.

Floresco SB, Block AE, Tse MT. 2008. Inactivation of the medial prefrontal cortex of the rat impairs strategy set-shifting, but not reversal learning, using a novel, automated procedure. Behav Brain Res 190: 85-96.

Granon S, Vidal C, Thinus-Blanc C, Changeux JP, Poucet B. 1994. Working memory, response selection, and effortful processing in rats with medial prefrontal lesions. Behav Neurosci 108: 883-891.

Hirsh R. 1974. The hippocampus and contextual retrieval of information from memory: A theory. Behav Biol 12: 421-444.

Hoover WB, Vertes RP. 2007. Anatomical analysis of afferent projections to the medial prefrontal cortex in the rat. Brain Struct Funct 212: 149-179.

Incisa della Rocchetta A, Milner B. 1993. Strategic search and retrieval inhibition: The role of the frontal lobes. Neuropsychologia 31: $503-524$.

Jo YS, Park EH, Kim IH, Park SK, Kim H, Kim HT, Choi JS. 2007. The medial prefrontal cortex is involved in spatial memory retrieval under partial-cue conditions. J Neurosci 27: 13567-13578.

Jonkman S, Mar AC, Dickinson A, Robbins TW, Everitt BJ. 2009. The rat prelimbic cortex mediates inhibitory response control but not the consolidation of instrumental learning. Behav Neurosci 123: 875-885.

Kuhl BA, Dudukovic NM, Kahn I, Wagner AD. 2007. Decreased demands on cognitive control reveal the neural processing benefits of forgetting. Nat Neurosci 10: 908-914.

Law LM, Smith DM. 2012. The anterior thalamus is critical for overcoming interference in a context-dependent odor discrimination task. Behav Neurosci 126: 710-719.

Lee I, Kesner RP. 2003. Time-dependent relationship between the dorsal hippocampus and the prefrontal cortex in spatial memory. J Neurosci 23: $1517-1523$

Lee I, Solivan F. 2008. The roles of the medial prefrontal cortex and hippocampus in a spatial paired-association task. Learn Mem 15: $357-367$.

Manns JR, Howard MW, Eichenbaum H. 2007. Gradual changes in hippocampal activity support remembering the order of events. Neuron 56: $530-540$

Miller EK, Cohen JD. 2001. An integrative theory of prefrontal cortex function. Annu Rev Neurosci 24: 167-202.

Monsell S. 2003. Task switching. Trends Cogn Sci 7: 134-140.

Munakata Y, Herd SA, Chatham CH, Depue BE, Banich MT, O'Reilly RC. 2011. A unified framework for inhibitory control. Trends Cogn Sci 15: 453-459.

Navawongse R, Eichenbaum H. 2013. Pathways that support memory retrieval in hippocampal neuronal ensembles. J Neurosci 33: $1002-1013$

Ng CW, Noblejas MI, Rodefer JS, Smith CB, Poremba A. 2007. Double dissociation of attentional resources: Prefrontal versus cingulate cortices. J Neurosci 27: 12123-12131.

Paxinos G, Watson C. 1998. The rat brain in stereotaxic coordinates. Academic Press, San Diego, CA.

Petrides M, Milner B. 1982. Deficits on subject-ordered tasks after frontal- and temporal-lobe lesions in man. Neuropsychologia 20: $249-262$.

Ragozzino ME. 2007. The contribution of the medial prefrontal cortex orbitofrontal cortex, and dorsomedial striatum to behavioral flexibility. Ann N Y Acad Sci 1121: 355-375.

Ragozzino ME, Detrick S, Kesner RP. 1999a. Involvement of the prelimbicinfralimbic areas of the rodent prefrontal cortex in behavioral flexibility for place and response learning. J Neurosci 19: 4585-4594.

Ragozzino ME, Wilcox C, Raso M, Kesner RP. 1999b. Involvement of rodent prefrontal cortex subregions in strategy switching. Behav Neurosci 113: $32-41$.
Ragozzino ME, Kim J, Hassert D, Minniti N, Kiang C. 2003. The contribution of the rat prelimbic-infralimbic areas to different forms of task switching. Behav Neurosci 117: 1054-1065.

Ranganath C, Cohen MX, Brozinsky CJ. 2005. Working memory maintenance contributes to long-term memory formation: Neural and behavioral evidence. J Cogn Neurosci 17: 994-1010.

Reber PJ, Siwiec RM, Gitelman DR, Parrish TB, Mesulam MM, Paller KA. 2002. Neural correlates of successful encoding identified using functional magnetic resonance imaging. J Neurosci 22: 9541-9548.

Rich EL, Shapiro ML. 2007. Prelimbic/infralimbic inactivation impairs memory for multiple task switches, but not flexible selection of familiar tasks. J Neurosci 27: 4747-4755.

Rubia K, Smith AB, Brammer MJ, Taylor E. 2003. Right inferior prefrontal cortex mediates response inhibition while mesial prefrontal cortex is responsible for error detection. NeuroImage 20: 351-358.

Seamans JK, Lapish CC, Durstewitz D. 2008. Comparing the prefrontal cortex of rats and primates: Insights from electrophysiology. Neurotox Res 14: $249-262$.

Shallice T, Burgess P. 1996. The domain of supervisory processes and temporal organization of behaviour. Philos Trans R Soc Lond B Biol Sci 351: 1405-1411; discussion 1411-1402.

Siapas AG, Lubenov EV, Wilson MA. 2005. Prefrontal phase locking to hippocampal theta oscillations. Neuron 46: 141-151.

Smith EE, Jonides J. 1999. Storage and executive processes in the frontal lobes. Science 283: 1657-1661.

Smith DM, Mizumori SJ. 2006. Hippocampal place cells, context, and episodic memory. Hippocampus 16: 716-729.

Smith ML, Leonard G, Crane J, Milner B. 1995. The effects of frontal- or temporal-lobe lesions on susceptibility to interference in spatial memory. Neuropsychologia 33: 275-285.

Sotres-Bayon F, Quirk GJ. 2010. Prefrontal control of fear: More than just extinction. Curr Opin Neurobiol 20: 231-235.

Spaniol J, Davidson PS, Kim AS, Han H, Moscovitch M, Grady CL. 2009. Event-related fMRI studies of episodic encoding and retrieval: Meta-analyses using activation likelihood estimation. Neuropsychologia 47: $1765-1779$.

Swanson LW. 1981. A direct projection from Ammon's horn to prefrontal cortex in the rat. Brain Res 217: 150-154.

Tamm L, Menon V, Reiss AL. 2002. Maturation of brain function associated with response inhibition. J Am Acad Child Adolesc Psychiatry 41: 1231-1238.

Uylings HB, Groenewegen HJ, Kolb B. 2003. Do rats have a prefrontal cortex? Behav Brain Res 146: 3-17.

Verbruggen F, Logan GD. 2008. Response inhibition in the stop-signal paradigm. Trends Cogn Sci 12: 418-424.

Vertes RP. 2006. Interactions among the medial prefrontal cortex, hippocampus and midline thalamus in emotional and cognitive processing in the rat. Neuroscience 142: 1-20.

Wagner AD, Pare-Blagoev EJ, Clark J, Poldrack RA. 2001. Recovering meaning: Left prefrontal cortex guides controlled semantic retrieval. Neuron 31: 329-338.

Wilson A, Brooks DC, Bouton ME. 1995. The role of the rat hippocampal system in several effects of context in extinction. Behav Neurosci 109: $828-836$

Wiltgen BJ, Brown RA, Talton LE, Silva AJ. 2004. New circuits for old memories: The role of the neocortex in consolidation. Neuron 44: $101-108$

Wimber M, Bauml KH, Bergstrom Z, Markopoulos G, Heinze HJ, Richardson-Klavehn A. 2008. Neural markers of inhibition in human memory retrieval. J Neurosci 28: 13419-13427.

Wood ER, Dudchenko PA, Eichenbaum H. 1999. The global record of memory in hippocampal neuronal activity. Nature 397: 613-616.

Received November 1, 2012; accepted in revised form January 18, 2013. 


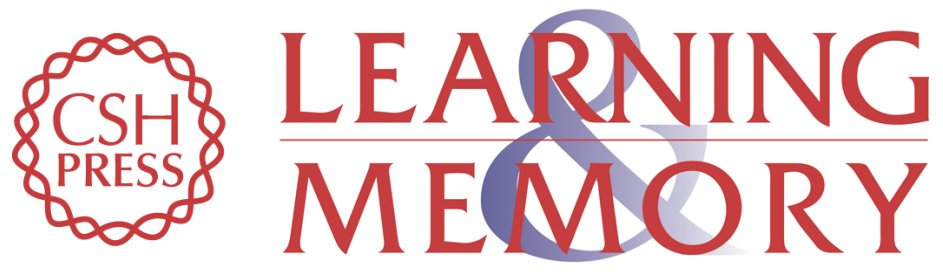

\section{The medial prefrontal cortex is critical for memory retrieval and resolving interference}

Gregory J. Peters, Christopher N. David, Madison D. Marcus, et al.

Learn. Mem. 2013, 20:

Access the most recent version at doi:10.1101/Im.029249.112

References This article cites 66 articles, 15 of which can be accessed free at:

http://learnmem.cshlp.org/content/20/4/201.full.html\#ref-list-1

License

Email Alerting Receive free email alerts when new articles cite this article - sign up in the box at the Service top right corner of the article or click here. 